\title{
Doppler tomography of the dwarf novae VY Aquari and WX Ceti ${ }^{\star}$
}

\author{
R. E. Mennickent ${ }^{1}$, E. Unda-Sanzana ${ }^{2}$, and C. Tappert ${ }^{3}$ \\ 1 Universidad de Concepción, Departamento de Física, Casilla 160-C, Concepción, Chile \\ e-mail: rmennick@astro-udec.cl \\ 2 Universidad Católica del Norte, Avenida Angamos 610, Antofagasta, Chile \\ e-mail: eundas@almagesto.org \\ 3 Departamento de Astronomía y Astrofísica, Pontificia Universidad Católica, Casilla 306, Santiago 22, Chile \\ e-mail: ctappert@astro.puc.cl
}

Received 12 January 2006 / Accepted 22 February 2006

\section{ABSTRACT}

Aims. We present the optical spectroscopy of the dwarf novae VY Aqr and WX Cet in quiescence.

Methods. For the first time for VY Aqr, we construct the Doppler tomograms for the H $\alpha, \mathrm{H} \beta$, and He I $5875 \AA$ A emission lines.

Results. The tomograms reveal complex emission structures that can be identified with the accretion disc, the bright spot and, in the case of VY Aqr, the secondary star. For the first time, the white dwarf is detected unambiguously in the spectrum of VY Aqr, and we confirm the long-lasting condition of the emission spot in the leading side of the WX Cet disc. For VY Aqr, we found that the bright spot influences the H $\alpha$ emission even at the large velocities far from the line center, introducing a spurious effect in the diagnostic diagram and ephemeris zero point. We calculate averaged $\mathrm{H} \alpha$ emissivity gradients in the discs and disc sizes and compare our results to previous investigations on these objects.

Key words. accretion, accretion disks - stars: dwarf novae - stars: individual: VY Aquari - stars: individual: WX Ceti

\section{Introduction}

Dwarf novae are binary systems consisting of a Roche-lobefilling secondary star transferring matter onto a non-magnetic white dwarf. In the process of mass-transfer, an accretion disc is formed around the white dwarf, releasing gravitational energy in the form of radiation. The disc contributes to the overall optical luminosity of the system with a luminosity that is usually larger than that of the white dwarf and of cool secondary star. In some short-period systems, however, especially those characterized by very low mass-transfer rates, the disc is faint, and the white dwarf can be visible in the spectrum. These systems are important, since they can be used as tracers of the dwarf novae population through the determination or the constraint of fundamental parameters like stellar mass and orbital parameters. Stellar masses for secondary stars, for instance, are poorly constrained among short-period dwarf novae (Patterson 2001). Furthermore, although dwarf novae have been studied for decades (for a review see Warner 1995), subtle details of the mass transfer process remain obscure. Prominent examples include the nature of the spiral waves seen in the disc during outburst and the role of secondary stars and their tidal forces on gas dynamics (Boffin et al. 2001).

* Based on observations collected at the European Southern Observatory, Chile. ESO programme 61.D-0396.
In this paper, we present spectroscopic data of VY Aqr and WX Cet, two short orbital period dwarf novae matching the characteristics mentioned above, as well as an analysis of the spectra using the Doppler tomography technique. We compare our results to previously published data for these sources and discuss them in the context of accretion discs in short orbital period dwarf novae and their long-term evolution.

\section{Observations and their reduction}

Medium-resolution spectroscopic observations were taken in August 1998 at La Silla, using the 3.5-m New Technology Telescope (NTT) in conjunction with the ESO Multi-Mode Instrument (EMMI). All observations were taken with CCD 36, grating 8 , and a mean exposure time of $300 \mathrm{~s}$. The mean dispersion was $1.26 \AA$ pixel $^{-1}$, and the resolution about $3 \AA$. Data reductions were done in the usual way, using IRAF routines for de-biasing and flat-fielding images, and for extracting one-dimensional spectra and calibrating wavelength and flux. For this last step, the spectrophotometric standard star LTT 7379 was observed with a wide slit. While the typical rms of wavelength-calibration functions was $0.4 \AA$, the flux calibrations are expected to be accurate to $0.1 \mathrm{mag}$. A summary of the observations is given in Table 1 . 
Table 1. Summary of the data used in the analysis. $N$ is the number of spectra collected per night.

\begin{tabular}{lccccc}
\hline \hline Object & $\begin{array}{c}\text { Date } \\
(\mathrm{UT})\end{array}$ & $\begin{array}{c}\text { Start-End } \\
(\mathrm{UT})\end{array}$ & $\begin{array}{c}\text { Orbits } \\
\text { covered }\end{array}$ & $\begin{array}{c}\lambda \text { range } \\
(\AA)\end{array}$ & $N$ \\
\hline WX Cet & 29/30 Aug. 1998 & $06: 53-10: 03$ & 2.26 & $4474-7038$ & 27 \\
WX Cet & 30/31 Aug. 1998 & $05: 01-06: 12$ & 0.85 & $4474-7038$ & 11 \\
VY Aqr & 30/31 Aug. 1998 & $02: 25-04: 35$ & 2.08 & $4474-7038$ & 20 \\
\hline
\end{tabular}

\section{WX Cet}

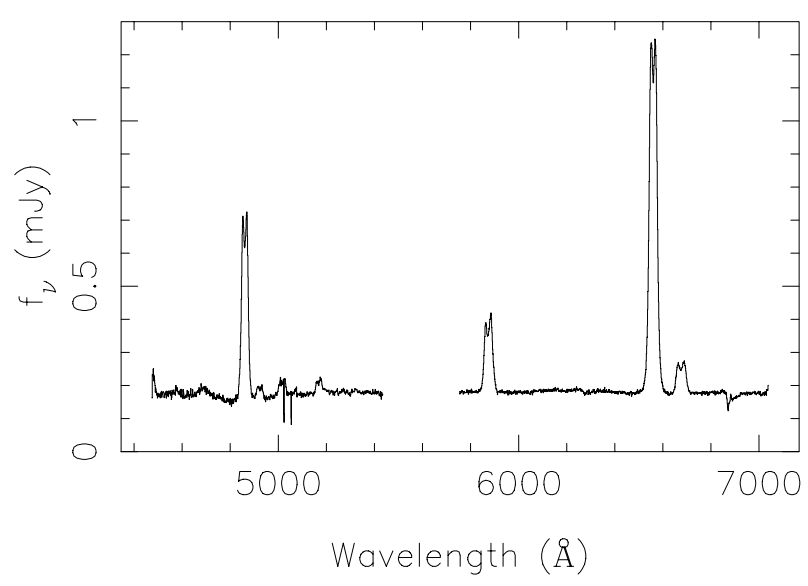

Fig. 1. Average flux-calibrated spectrum for WX Cet. The central section presented cosmetic defects and was removed from the analysis.

Five images bracketing our spectroscopic observations were obtained by VSNET observers (Kato et al. 2004) on August 28-30 and on September 2 and 4. They indicate that VY Aqr was observed in quiescence, with a $V$ magnitude fainter than 14 .6. VSNET images obtained on August 24 and September 2 show WX Cet in quiescence, too, with a $V$ magnitude fainter than $\sim 14$.

\section{Results}

\subsection{Average spectra}

Figures 1 and 2 show the average spectra for WX Cet and VY Aqr. In both figures, some absorption artifacts are seen redward of $5000 \AA$. These were introduced by cosmetic problems on the CCD and could not be accounted for during the reduction process. Consequently, they were excluded from the analysis. The spectral absorption feature redward of $6800 \AA$ corresponds to the telluric $B$ band.

Both VY Aqr and WX Cet show broad Balmer and helium emission lines. The strongest are $\mathrm{H} \alpha, \mathrm{H} \beta$, and He I $5875.618 \AA$, although He I $6678.149 \AA$ and some weak Fe II lines are also noticeable (4923.92, 5018.44, and 5169.03 $\AA$ ).

The profiles show a double-peaked structure that is commonly seen in hydrogen-dominated CVs. This is usually explained by the presence of a rotating accretion disc around the mass accretor in a binary system.

The white dwarf absorption is clearly seen around the $\mathrm{H} \beta$ emission of VY Aqr, indicating a very faint disc. In the spectra of Thorstensen \& Taylor (1997), this feature was only

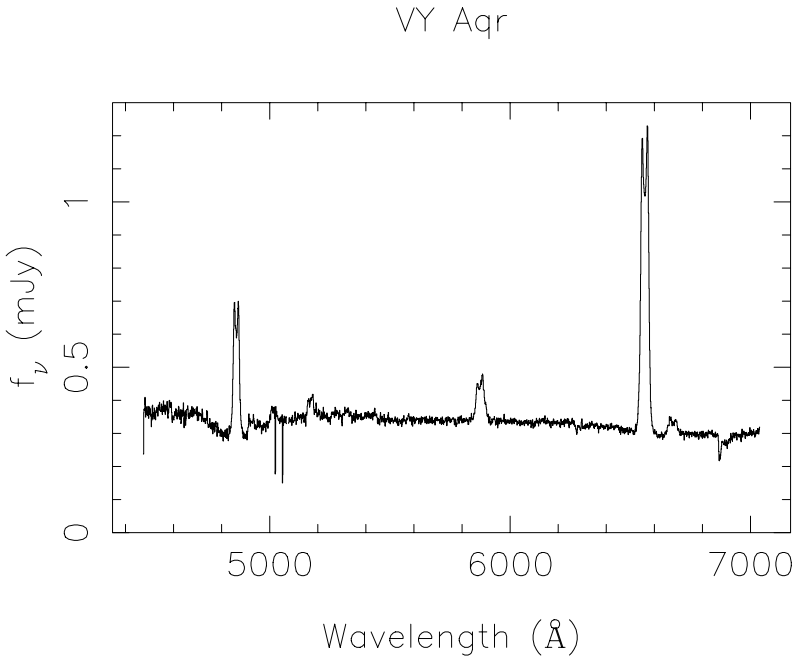

Fig. 2. Average flux-calibrated spectrum for VY Aqr.

marginally visible. For WX Cet, the smooth depression around the $\mathrm{H} \beta$ emission suggests the presence of a white dwarf in the spectrum, but the spectral $\mathrm{S} / \mathrm{N}$ is not high enough to make a conclusive statement about this point.

We measured the peak separation $(\Delta \lambda)$ and other line widths for the main emission lines in the averaged spectra. These data are given in Table 2 . We defined $A_{84} \equiv \log W_{04}$ $\log W_{08}$ and $A_{41} \equiv \log W_{01}-\log W_{04}$ in the main emission lines, where $W_{0 n}$ represents the line width at a fraction of $n \times 10 \%$ its maximum height above the continuum. Using well-defined basis assumptions, the parameters $A_{84}$ and $A_{41}$ are good indicators of the disc line emissivity and the inner disc radius. Assuming an axially symmetric, flat, Keplerian disc and a line emissivity $\propto r^{-\alpha}$, Smak (1981) found a relationship between the pairs $A_{84}-A_{41}$ and $\alpha-R$, where $R \equiv r_{\text {in }} / r_{\text {out }}$ is the ratio between the inner and outer disc radius. The mapping function turned out to be only weakly dependent on the instrumental resolution.

Figure 3 shows our results along with theoretical isoparametric curves from Smak (1981). In this figure, we also include the data for SU UMa stars given by Mennickent \& Arenas (1998) and the position of 1RXS J105010.3-140431, a candidate for WZ Sge-type dwarf novae (Mennickent et al. 2001). We found that the $\mathrm{H} \alpha A_{84}-A_{41}$ pair for WXCet $\left(A_{84}=\right.$ $0.14 \pm 0.01$ and $\left.A_{41}=0.16 \pm 0.01\right)$ practically coincides with that found by Mennickent \& Arenas (1998) in spectra taken in 1992 (viz. $0.15 \pm 0.04$ and $0.17 \pm 0.04$ ). In addition, the relative emission widths for different lines of this star are quite similar. From the position of the $\mathrm{H} \alpha$ data point in the plot, we deduce $\alpha=1.7$ and $R=0.15$. On the other hand, for VY Aqr, the $\mathrm{H} \alpha$ emission is compatible with an emissivity exponent $\alpha=1.5$ and 
Table 2. Line width parameters for different emission lines in the averaged spectra. For WX Cet, the maximum intensity was measured both in the blue and red peaks of the asymmetric He I profile. For VY Aqr, the $\mathrm{H} \beta$ emission line widths are probably affected by the underlying white dwarf absorption. The error in the last digit is given in parenthesis.

\begin{tabular}{lccccc}
\hline \hline Object & Line & $\Delta \lambda\left(\mathrm{km} \mathrm{s}^{-1}\right)$ & $\mathrm{A}_{84}$ & $\mathrm{~A}_{41}$ & Note \\
\hline VY Aqr & $\mathrm{H} \alpha$ & 980 & $0.11(1)$ & $0.12(1)$ & \\
VY Aqr & $\mathrm{H} \beta$ & 980 & $0.10(1)$ & $0.10(1)$ & doubtful \\
VY Aqr & He I 5875 & 1060 & $0.20(1)$ & $0.16(1)$ & \\
WX Cet & $\mathrm{H} \alpha$ & 725 & $0.14(1)$ & $0.16(1)$ & \\
WX Cet & $\mathrm{H} \beta$ & 960 & $0.15(1)$ & $0.12(1)$ & \\
WX Cet & He I 5875 & 1080 & $0.13(1)$ & $0.14(1)$ & max. blue peak \\
WX Cet & He I5875 & 1080 & $0.15(1)$ & $0.15(1)$ & max. red peak \\
\hline
\end{tabular}

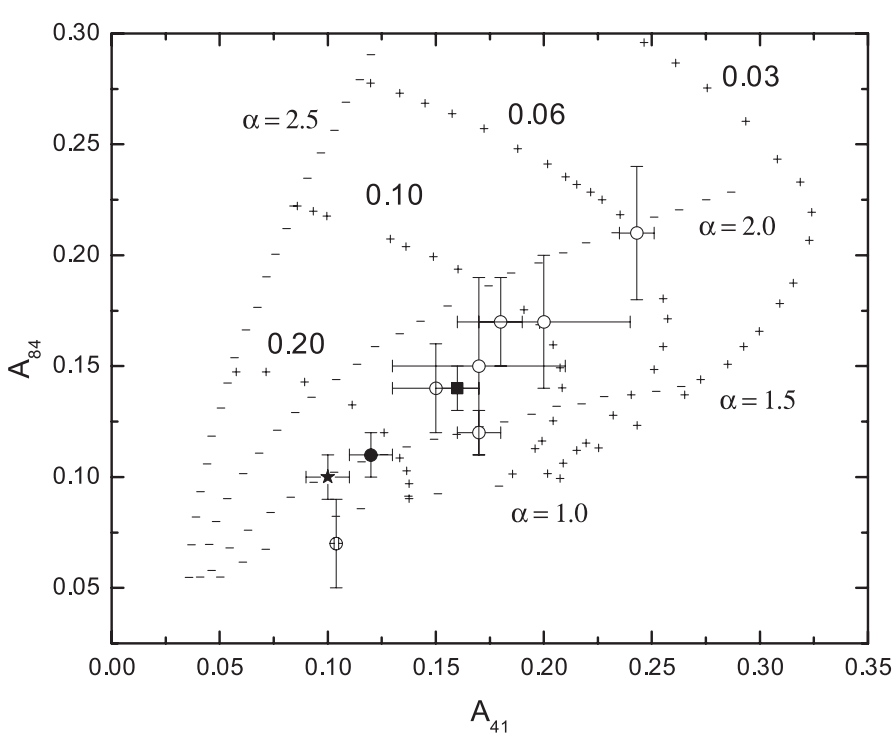

Fig. 3. The $A_{84}-A_{41}$ diagram for VYAqr (filled circle) and WXCet (filled square), along with the SUUMa stars listed by Mennickent \& Arenas (1998, open circles) and 1RXS J105010.3-140431 (Mennickent et al. 1991, star). Only H $\alpha$ values of quiescence dwarf novae have been plotted. Isoparametric curves are labeled with their $\alpha$ and $R$ values according to Smak (1981).

$R=0.22$. The discrepancy observed with the He I 5875 data in Table 2 is probably due to the fact that almost all emission in this line arises from the bright spot (see next section) in clear contradiction to the assumption of axial symmetry. Indeed, the major problem with the above analysis stems from this assumption, since observations clearly indicate that the emissivity is also a function of the azimuthal angle of the disc (see Doppler maps in the next section). In addition, some results indicate that orbits could not be Keplerian in certain dwarf nova discs (Martinez-Pais et al. 1994; Marsh \& Horne 1990). However, we can still interpret our results as a measure of the averaged emissivity in the azimuthal direction and consider that the results are less-biased in the cases where most emission comes from the disc and not from the spots. The Smak method, although not validated in its basic assumptions by modern observations, still remains a useful tool to compare the accretion discs of different systems. We observe in Fig. 3 that the dwarf novae occupy a well-defined space in the diagram, a
Table 3. Results of the diagnostic diagram and comparison to previously published data. "NA" means not available.

\begin{tabular}{lccc}
\hline \hline Object & Source & $\gamma\left(\mathrm{km} \mathrm{s}^{-1}\right)$ & $K\left(\mathrm{~km} \mathrm{~s}^{-1}\right)$ \\
\hline VY Aqr & This paper & $-118 \pm 5$ & $100 \pm 7$ \\
VY Aqr & Thorstensen \& Taylor (1997) & $-25 \pm 3$ & $49 \pm 4$ \\
WX Cet & This paper & $-131 \pm 7$ & $27 \pm 10$ \\
WX Cet & Tappert et al. (2003) & NA & $59 \pm 18$ \\
\hline
\end{tabular}

fact already noted by Mennickent \& Arenas (1998). The figure can be interpreted assuming that discs of long recurrencetime dwarf novae have flatter emissivity distributions than those of dwarf novae with shorter recurrence times. They also have lower $R$ values, which was interpreted by Mennickent \& Arenas (1998) as evidence that long supercycle-length dwarf novae have truncated discs. The position of VY Aqr and WXCet is rather intermediate, between the extremely long-recurrence time dwarf nova WZ Sge and the WZ-Sge type candidate 1RXS J105010.3-140431 (the two lower points in the figure) and the other dwarf novae in the sample.

\subsection{Doppler tomography}

The technique of Doppler tomography allows us to produce a map summarizing all the information on the respective emission lines in our data sets. This map is plotted in velocity coordinates that are not translated into position coordinates because the corresponding transformation law is not known a priori for all emission sources. Although the maps are consequently interpreted using this representation in velocity, it is possible to isolate some constituents of the binary system and to compare them to models. For standard references on Doppler tomography, see Marsh \& Horne (1988) and Marsh (2001).

To determine the physical orientation of the maps, we searched for an ephemeris for VY Aqr and WX Cet based on our spectroscopic data set, using the double-Gaussian technique for measuring radial velocities (Schneider \& Young 1980) and the popular analysis method of the line diagnostic diagram (Shafter 1983). This method provides the best solution for the line $\gamma$ velocity, radial velocity half-amplitude $K$, and zero phase, exploring the dynamics of the emission line profile at different velocity sections during the binary orbit. The method calculates the centroid of the emission line, convolving it with two Gaussians of fixed width and variable separation $d$. 


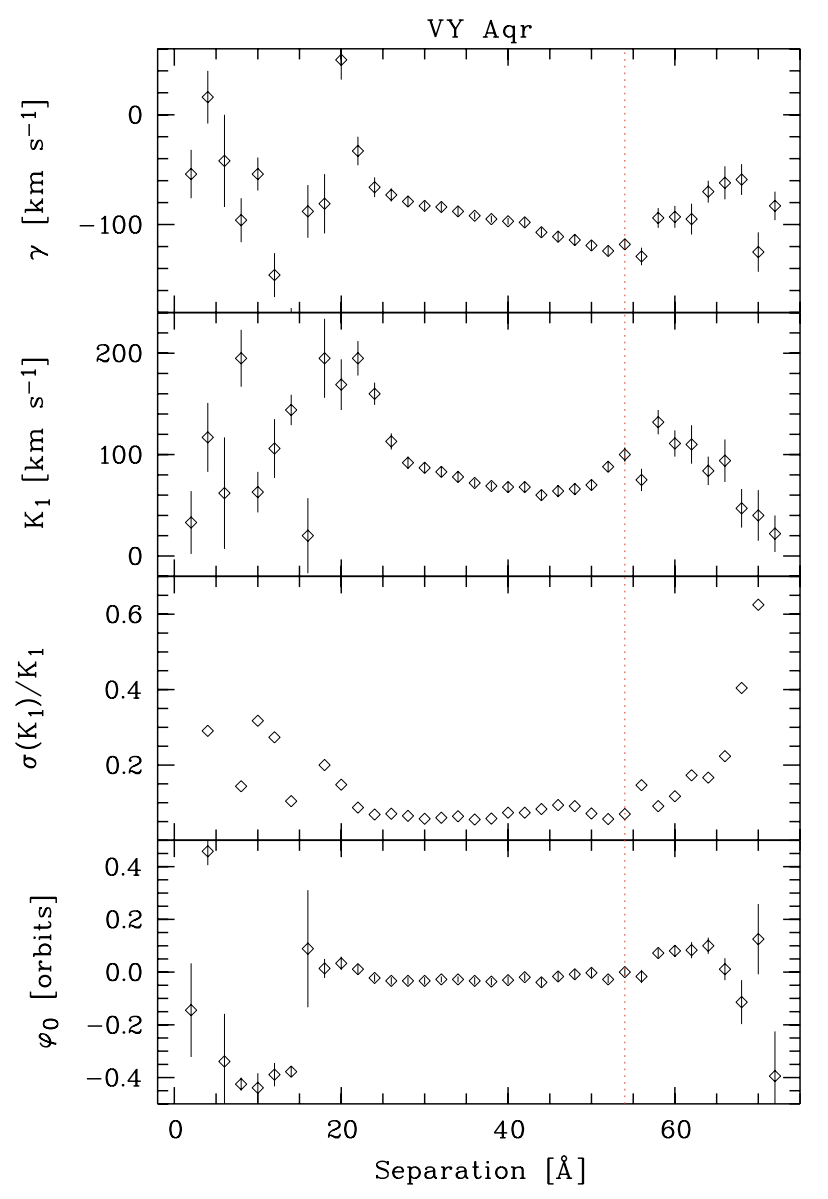

Fig. 4. The diagnostic diagram for VY Aqr. The dotted line marks the values for the most probable zero phase.

Different radial velocity curves are obtained for a range of $d$ values. The best solution corresponds to the last separation $d_{\max }$ before the noise begins to dominate the signal. In the diagnostic diagram, this point is ideally represented by a sharp increase in $\sigma_{K} / K$, where $\sigma_{K}$ is the $K$ error. The diagnostic diagrams for the $\mathrm{H} \alpha$ emission line of VY Aqr and WX Cet are shown in Figs. 4 and 5 . We observe that the best solutions reveal a radial velocity half-amplitude of $100 \pm 7 \mathrm{~km} \mathrm{~s}^{-1}$ and $27 \pm 10 \mathrm{~km} \mathrm{~s}^{-1}$, a $\gamma$ velocity of $-118 \pm 5 \mathrm{~km} \mathrm{~s}^{-1}$ and $-131 \pm 7 \mathrm{~km} \mathrm{~s}^{-1}$, and a zero phase of HJD $=2451055.5941 \pm 0.0009$ and HJD $=$ $2451054.7683 \pm 0.0037$ for VY Aqr and WX Cet, respectively. These $K$ values differ from those published by Thorstensen $\&$ Taylor (1997) and Tappert et al. (2003) (see Table 3). In the case of VY Aqr, the choice for the best separation is somewhat ambiguous: if one considers the increase in noise at $d_{\max }=56 \AA$ as insignificant, then $d=64 \AA$ appears to be the next best separation. However, the corresponding radial velocity parameters still deviate significantly from those found by Thorstensen \& Taylor (1997). Since the H $\alpha$ Doppler map results in a nonstandard position for key features of a CV, like the bright spot region, we suspect that our ephemeris suffers some kind of perturbation and is probably biased. One possible explanation for this mismatch is that we did not reach the wings of the emission profiles with $\mathrm{S} / \mathrm{N}$ high enough to detect the true best solution for the zero phase and the other dynamical parameters.

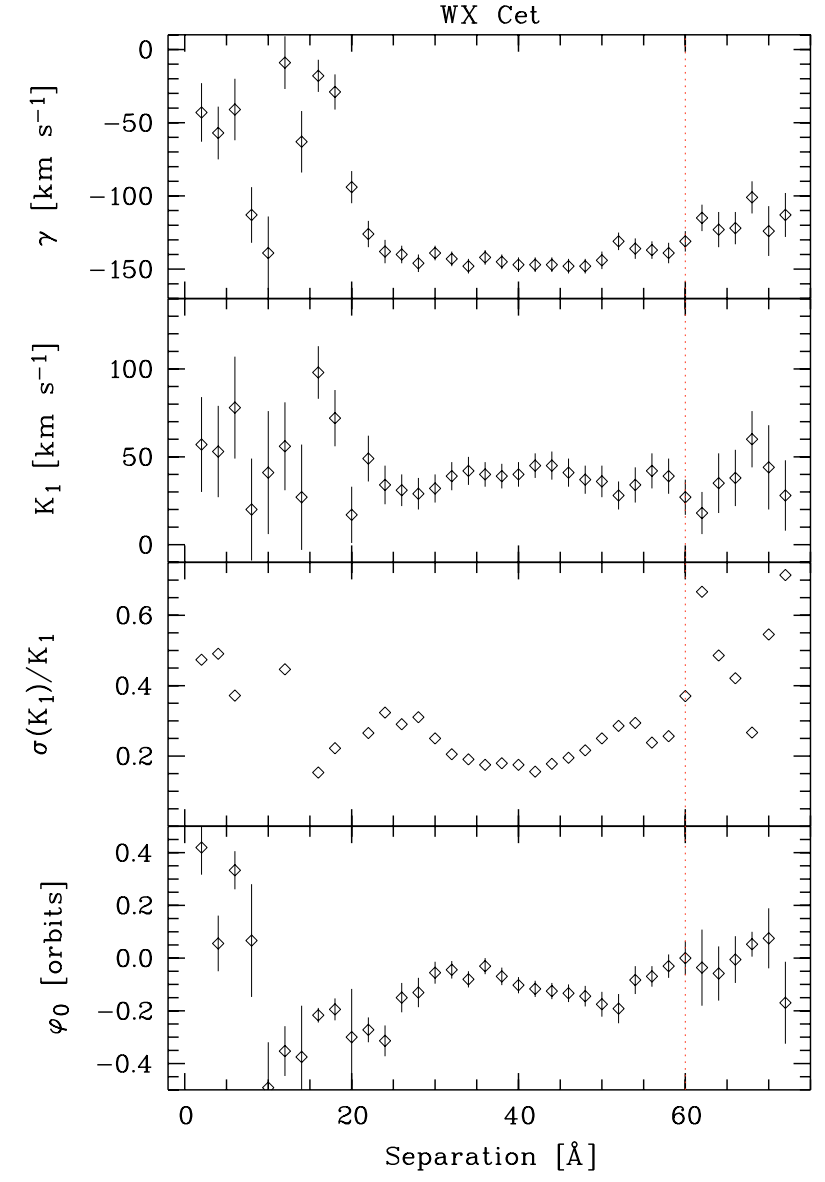

Fig. 5. The diagnostic diagram for WX Cet. The dotted line marks the values for the most probable zero phase.

Another explanation is that the bright spot component influences the measurements made on the emission profiles, even at high velocities, so that the diagnostic method probes, in this case, the bright spot rather than the disc motion. After a careful examination of the trailed spectrograms at different intensity levels, we concluded that this last interpretation is most probably the correct one (Fig. 6). Similar effects have been observed in other dwarf novae (e.g. Billington et al. 1996), and one explanation could be the Stark broadening operating in the bright spot region. Note that Stark broadening has been suggested as a possible mechanism operating in discs of cataclysmic variable stars (Lin et al. 1998).

In Fig. 7, we present the tomograms obtained for the strongest lines in our datasets. In the case of VY Aqr, the map for $\mathrm{H} \alpha$ displays a weak extended spot on the inner side of a large ring. We understand this ring (see also $\mathrm{H} \beta$ ) to be the signature of the accretion disc and the extended spot to be the secondary star. Assuming this interpretation to be correct, we introduced an offset of 0.2 orbital phases, with respect to the value extracted from the diagnostic diagram, to the maps, to put the secondary star centered in the vertical axis, which is the predicted location for a $\mathrm{CV}$ rotating as a rigid body. This also fixes the location of a strong region of emission inside the ring (see He I, in particular), which we understand to be coming from the point of impact of the gas stream on the disc, usually called the bright spot. 

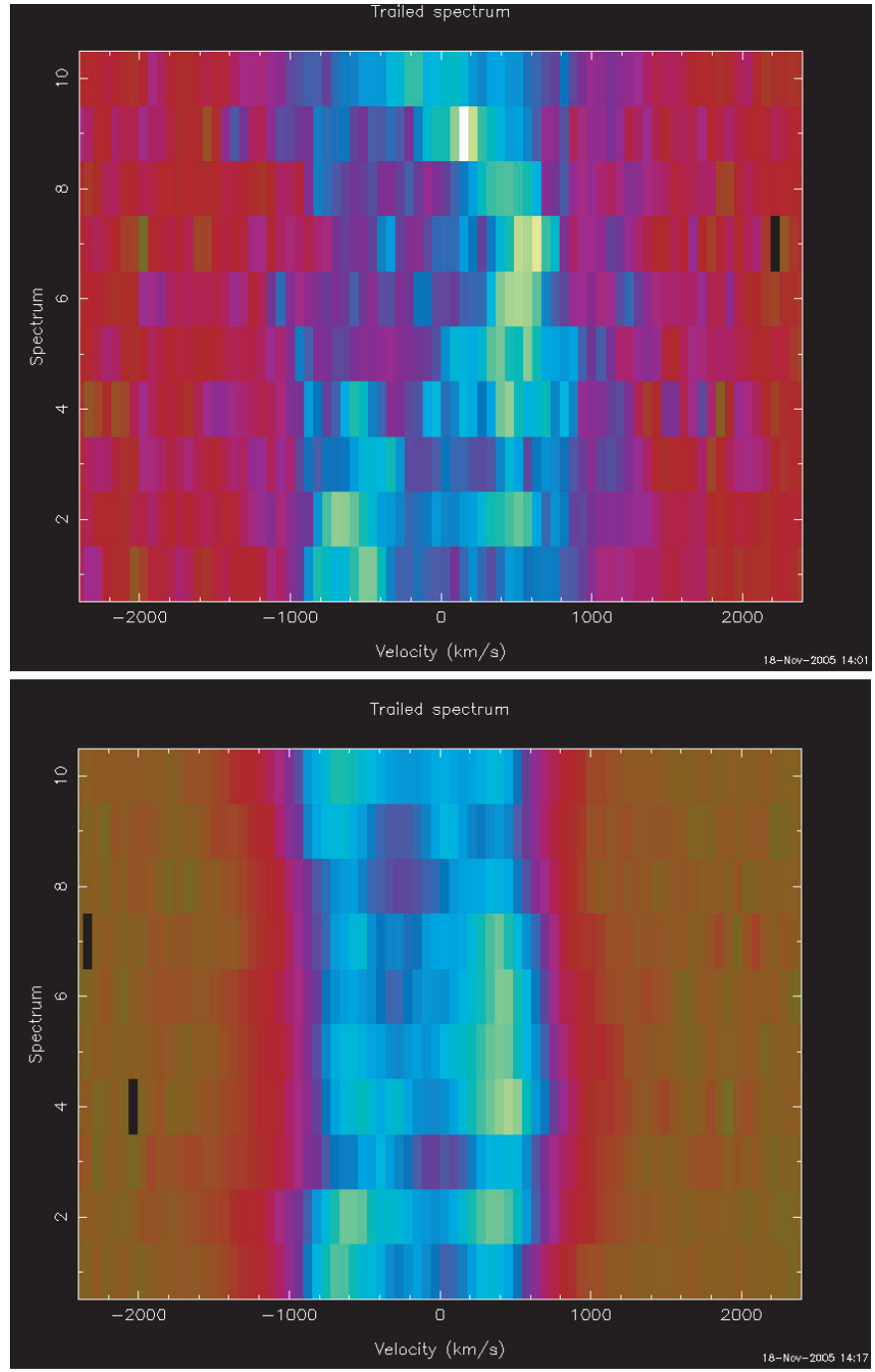

Fig. 6. The trailed spectra for $\mathrm{He} \mathrm{I} \lambda 5875$ (top) and $\mathrm{H} \alpha$ (bottom), showing the influence of the bright spot on the emission wings. The phases go from +0.5 to -0.5 , from top to bottom, in both diagrams.

For WX Cet, we introduced the same offset of 0.2 orbital phases, but in this case the procedure is less well-justified. The secondary star is not immediately obvious in any of the lines, and thus our only guide for the correction is the location of the bright spot. Other features are similar to what we described for VY Aqr.

\section{Discussion}

A Doppler map for VY Aqr was published by Littlefair et al. (2000), based on spectra of the infrared Paschen- $\beta$ line. This Doppler map shows a faint ring corresponding to disc emission and a strong peak associated with the bright spot. Using an educated guess for the phase offset, these authors find that the bright spot in VY Aqr is located downstream from the stream impact region. Using the skew-mapping technique, these authors were also able to derive a value for the radial velocity semi-amplitude of the secondary star in VY Aqr of $K_{R}=$ $320 \pm 70 \mathrm{~km} \mathrm{~s}^{-1}$, which in conjunction with $K_{W}=49 \pm 4 \mathrm{~km} \mathrm{~s}^{-1}$ given by Thorstensen \& Taylor (1997), gives a mass ratio of $q=0.15 \pm 0.04$. In addition, from a model-fitting of IR spectra, Mennickent et al. (2004) presented evidence for a L $0 \pm 2$ secondary star in VY Aqr. If we use an upper limit of $0.08 M_{\odot}$ for this secondary star (Baraffe \& Chabrier 1996), we estimate a mass for the white dwarf of about $\leq 0.54 M_{\odot}$. This relatively low mass for the white dwarf is confirmed by Hamilton \& Sion (2004), who find the best fit to a IUE spectrum for VY Aqr (during outburst) to be a white dwarf mass of $0.55 M_{\odot}$, inclination of 41 degrees, and a scale-factor distance of 93 parsecs, which they note is in agreement with the parallax distance of $97 \pm 13$ pc found by Thorstensen (2003).

Our $\mathrm{H} \alpha$ map shows the disc emission, as well as emission from the bright spot. The $\mathrm{H} \beta$ map shows similar features, although in this case the bright spot is more luminous than the rest of the disc, and there is another emission spot in the third quadrant (quadrants are usually counted counterclockwise, starting from the upper right quadrant). He I 5875 gives stronger emissions in the bright spot than from the rest of the disc. This finding is probably related to the high temperature in the bright spot region when compared to the temperature of the surrounding disc. The $\mathrm{H} \alpha$ map clearly reveals the secondary star (Figs. 7 and 8).

Doppler maps for WX Cet were first constructed by Tappert et al. (2003). These authors observed a spotty structure in the $\mathrm{H} \alpha$ map of WX Cet and no sign of the accretion disc, and they interpreted the primary as an artifact produced by the noisy data. In addition, they observed a recurrent emission on the leading side of the map (i.e. those quadrants with $v x>0$ ) in all their individual data sets, and concluded that this was a real feature. Our data set is of much better quality than that of Tappert et al. (2003), as can be observed in Fig. 7, and the disc is clearly seen in $\mathrm{H} \alpha$ and $\mathrm{H} \beta$. This fact supports the interpretation by Tappert et al. (2003) about the spotty structure of their maps, i.e. that noisy data are the reason we cannot clearly see the disc shape. On the other hand, the enhanced emission in the leading side observed by Tappert et al. (2003) is still present in our $\mathrm{H} \alpha$ map, confirming that is a real feature and indicating a persistent condition. Mennickent (1994) also found evidence for an enhanced emission region on the leading side of the disc, but based his conclusion on the behaviour of the $\mathrm{H} \alpha$ emission line. However, surprisingly, the same structure is not seen in the $\mathrm{H} \beta$ map. Emission arcs are visible in the second and third quadrants and are similar, but less spotty, than those seen in VY Aqr. The first of these arcs is possibly related to the bright spot. We also observe in Fig. 7 that the He I 5875 emission, although distributed over the disc, is much more concentrated in the bright spot region, as is the case for VY Aqr.

While we have detected the secondary star in VY Aqr, it is not unambiguously present in the Doppler maps of WX Cet, although it could be present in the $\mathrm{H} \alpha$ map at our detection threshold. In Fig. 7, there appears to be an emission at the same location as in VY Aqr, but it is not clear if this is part of the accretion disc or of another structure. We need data of better quality to resolve this question. It is not clear why the secondary is more visible in VY Aqr, since most evidence indicates very similar properties for these dwarf novae. Theoretically, the orbital periods (0.063 days for VY Aqr and 0.058 days for 


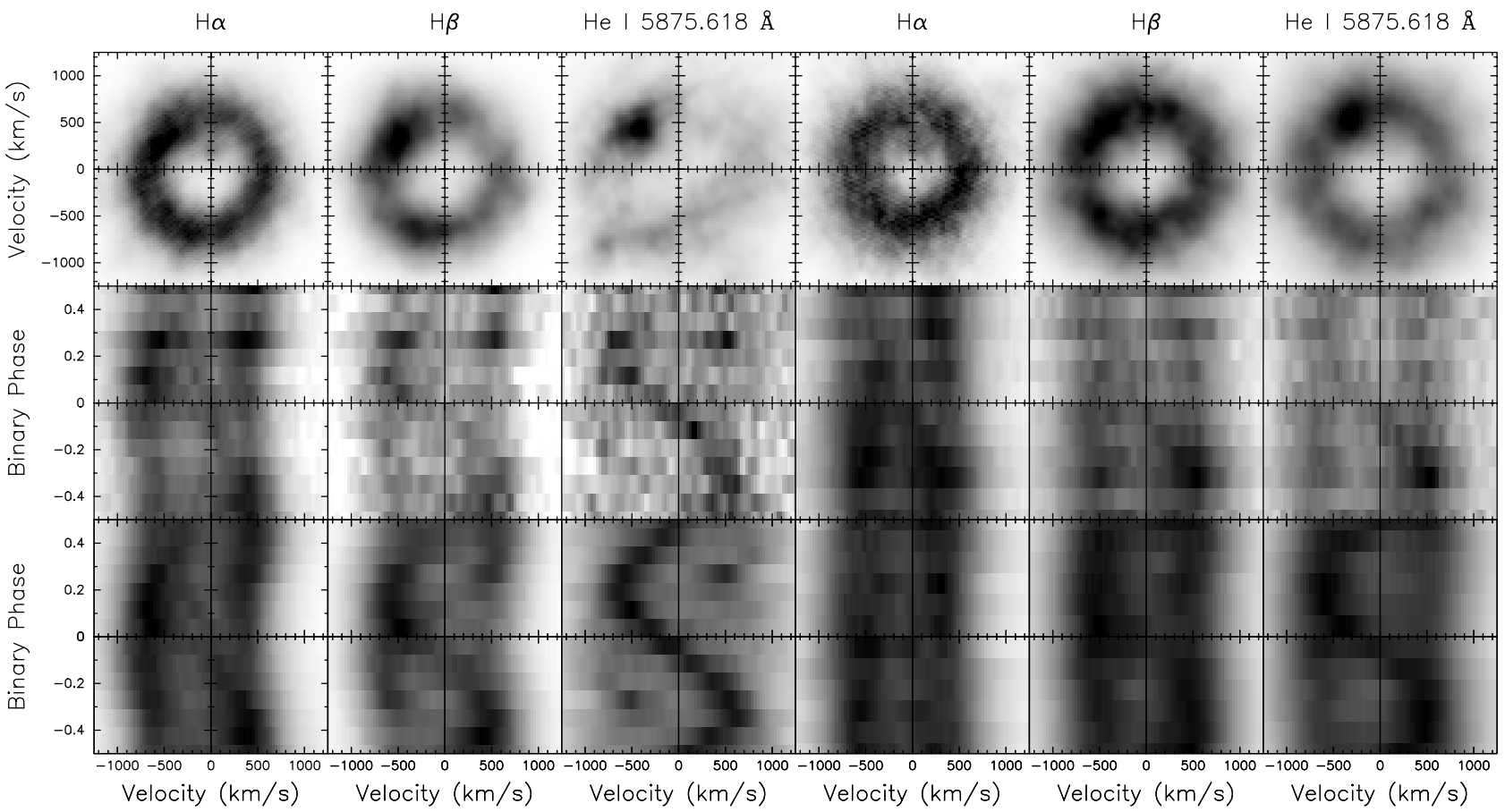

Fig. 7. Doppler tomography for VY Aqr (Cols. 1 to 3, from left to right) and WX Cet (Cols. 4 to 6). From top to bottom the rows show: 1) the Doppler tomograms, 2) a phase-binned template drawn from the observed spectra from which the tomograms were computed, and 3) data computed from the tomograms. The velocity scales are relative to the line centers. All grey-scales start from zero with the uppermost level saturated at $90 \%$ of the peak flux for the first row, and saturated evenly at $100 \%$ of the peak flux for the remaining rows.

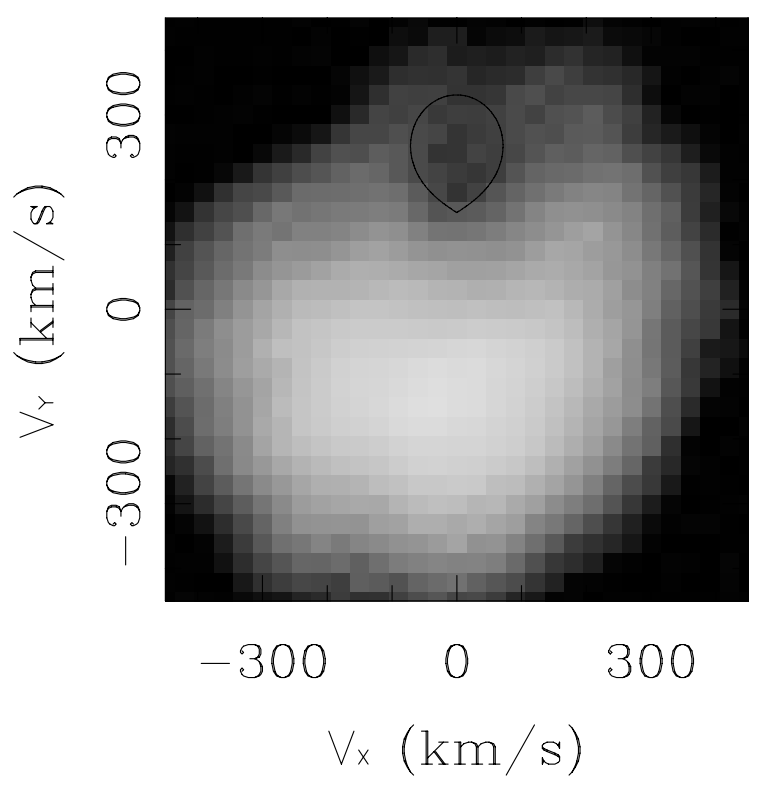

Fig. 8. A zoom around the zero velocity region of the VY Aqr $\mathrm{H} \alpha$ map, including an outline of the Roche lobe for the secondary star. The saturation is at $60 \%$ of the peak flux level. The structure seen inside the Roche lobe is contained in the range $40-50 \%$ of the peak flux level.

WX Cet) suggest similar mass transfer rates, whereas the recurrence times of the order of hundreds of days indicate very low mass accretion-rates and disc luminosities, for both. On the other hand, space-based observations suggest white dwarf temperatures between 13000 and $13500 \mathrm{~K}$ for both stars (Sion et al. 2003). The above suggests the same intensity of irradiating photons for both systems. In addition, the similar massratio derived from the superhump period (Patterson et al. 2005), indicates a similar secondary star mass. The above scenario conflicts with the detection of the secondary in only one of the systems, although as explained before, low $\mathrm{S} / \mathrm{N}$ spectra for WX Cet could be the reason for that conflict. Mason (2001), based on the observation and modeling of the spectral energy distribution, claims that WX Cet harbors a substellar secondary with a mass lower than 0.07 solar masses, while infrared spectroscopy indicates that VY Aqr has a secondary star near the upper branch of the $\mathrm{CV}$ evolution track (Mennickent et al. 2004). A substellar mass secondary for WX Cet is also suggested by the dynamic model of Rogoziecki \& Schwarzenberg-Czerny (2003). If this view is right, both stars should be at different evolutionary stages (Howell et al. 2001) and with secondaries at different temperatures, although when irradiated from the same source, the hotter one (VY Aqr) might be more easily visible than the cooler one (WX Cet).

The ratio of $\mathrm{H} \alpha$ and $\mathrm{H} \beta$ Doppler maps has been interpreted as a disc temperature map in some dwarf novae (e.g. Skidmore et al. 2000; Mennickent et al. 2001). The fact that we find different structures in our $\mathrm{H} \alpha$ and $\mathrm{H} \beta$ maps shows that in the case of VY Aqr and WX Cet, this ratio is not constant across the disc, suggesting density and temperature variations along the disc azimuthal direction. This empirical result conflicts with any potential theoretical disc model assuming steady-state conditions in quiescence. 


\section{Conclusions}

In this paper, we have constructed Doppler maps for the dwarf novae VY Aqr and WX Ceti. We have also provided a brief description and analysis of the spectrograms, getting insights on the structure of the accretion discs for these systems. We have confirmed the presence of an enhanced emission region at an anomalous location in WX Cet, which turns out to be a persistent feature, thus challenging any steady-state disc model. On the other hand, in a diagram of line emissivity and extension for the disc, VY Aqr and WX Cet occupy middle zones, compared to other dwarf novae with extreme properties (like WZ Sge and others with very short recurrence times). This fact suggests a link between the observable recurrence time, the disc size, and line emissivity, a fact already noted by Mennickent \& Arenas (1998). The discs of VY Aqr and WX Cet have flatter emissivity distributions than those of dwarf novae with shorter recurrence times. The fact that we have detected the secondary star in VY Aqr and not (unambiguously) in WX Cet, could be related to the different evolutionary stages of these systems. A definite conclusion about this point requires further study. Finally, we have found evidence that the bright spot contributes significantly to the $\mathrm{H} \alpha$ emission wings in both dwarf novae, perturbing the results of the diagnostic diagrams, especially in regards the ephemeris zero point. We caution future observers about the use and interpretation of the diagnostic diagram, since a test of influence of the high-velocity emission from the bright spot seems to be necessary to exclude any potential contamination in the line profile wings. In addition, we note that VYAqr and WXCet show evidence for the white dwarf in their spectra, so they are interesting candidates for future high-resolution, high- $\mathrm{S} / \mathrm{N}$ research aimed at resolving the white dwarf motion and calculating the binary parameters.

Acknowledgements. We acknowledge the referee, Dr. Littlefair, for helpful comments on a first version of this manuscript. R.E.M. acknowledges support by Fondecyt 1030707. C.T. acknowledges support by Fondecyt 1051078. E.U.S. acknowledges Tom Marsh for useful references for this work. The authors acknowledge the data analysis facilities provided by the Starlink Project. This research has also made use of NASA's Astrophysics Data System Bibliographic Services.

\section{References}

Baraffe, I., \& Chabrier, G. 1996, ApJ, 461, L51

Boffin, H. M., Steeghs, D., \& Cuypers, J., ed. 2001, Astrotomography: Indirect Imaging Methods in Observational Astronomy, Lecture Notes in Physics, 573

Billington, I., Marsh, T. R., Horne, K., et al. 1996, MNRAS, 279, 1274

Hamilton, R. T., \& Sion, E. M. 2004, PASP, 116, 926

Howell, S. B., Nelson, L. A., \& Rappaport, S. 2001, ApJ, 550, 897

Kato, T., Uemura, M., Ishioka, R., et al. 2004, PASJ, 56, 1

Lin, D. N. C., Williams, R. E., \& Stover, R. J. 1988, ApJ, 327, 234

Littlefair, S. P., Dhillon, V. S., Howell, S. B., \& Ciardi, D. R. 2000, MNRAS, 313, 117

Marsh, T. R. 2001, in Astrotomography, Lecture Notes in Physics, ed. H. Boffin, et al. (Springer), 573, 1

Marsh, T. R., \& Horne, K. 1988, MNRAS, 235, 269

Marsh, T. R., \& Horne, K. 1990, ApJ, 349, 593

Martinez-Pais, I. G., Giovannelli, F., Rossi C., \& Gaudenzi, S. 1994, A\&A, 291, 455

Mason, E. 2001, PhDT, Accretion disc Structure and Spectral Energy Distribution in Short Orbital Period Dwarf Novae, Planetary Science Institute

Mennickent, R. 1994, A\&A, 285, 979

Mennickent, R. E., \& Arenas, J. 1998, PASJ, 50, 333

Mennickent, R. E., Diaz, M., Skidmore, W., \& Sterken, C. 2001, A\&A, 376, 448

Mennickent, R. E., Diaz, M. P., \& Tappert, C. 2004, MNRAS, 347, 1180

Patterson, J. 2001, PASP, 113, 736

Patterson, J., Kemp, J., Harvey, D., et al. 2005, PASP, 117, 1204

Rogoziecki, P., \& Schwarzenberg-Czerny, A. 2003, A\&A, 397, 961

Shafter, A. W. 1983, ApJ, 267, 222

Schneider, D. P., \& Young, P. 1980, ApJ, 238, 946

Sion, E. M., Szkody, P., Cheng, F., Gänsicke, B. T., \& Howell, S. B. 2003, ApJ, 583, 907

Skidmore, W., Mason, E., Howell, S. B., et al. 2000, MNRAS, 318, 429

Smak, J. 1981, AcA, 31, 395

Tappert, C., Mennickent, R. E., Arenas, J., Matsumoto, K., \& Hanuschik, R. W. 2003, A\&A, 408, 651

Thorstensen, J. R. 2003, AJ, 126, 3017

Thorstensen, J. R., \& Taylor, C. J. 1997, PASP, 109, 1359

Warner, B. 1995, Cataclysmic Variable Stars (Cambridge University Press) 